\title{
Breast cancer PAM50 signature: correlation and concordance between RNA-Seq and digital multiplexed gene expression technologies in a triple negative breast cancer series
}

A. C. Picornell ${ }^{*}$, I. Echavarria², E. Alvarez', S. López-Tarruella ${ }^{3}$, Y. Jerez ${ }^{3}$, K. Hoadley $^{4}$, J. S. Parker ${ }^{4}$, M. del Monte-Millán ${ }^{3}$, R. Ramos-Medina ${ }^{3}$, J. Gayarre' ${ }^{3}$ I. Ocaña ${ }^{3}$, M. Cebollero ${ }^{5}$, T. Massarrah ${ }^{3}$, F. Moreno ${ }^{6}$, J. A. García Saenz ${ }^{6}$, H. Gómez Moreno ${ }^{7}$, A. Ballesteros ${ }^{8}$, M. Ruiz Borrego ${ }^{9}$, C. M. Perou ${ }^{10}$ and M. Martin ${ }^{11}$

\begin{abstract}
Background: Full RNA-Seq is a fundamental research tool for whole transcriptome analysis. However, it is too costly and time consuming to be used in routine clinical practice. We evaluated the transcript quantification agreement between RNA-Seq and a digital multiplexed gene expression platform, and the subtype call after running the PAM50 assay in a series of breast cancer patients classified as triple negative by IHC/FISH. The goal of this study is to analyze the concordance between both expression platforms overall, and for calling PAM50 triple negative breast cancer intrinsic subtypes in particular.

Results: The analyses were performed in paraffin-embedded tissues from 96 patients recruited in a multicenter, prospective, non-randomized neoadjuvant triple negative breast cancer trial (NCT01560663). Pre-treatment core biopsies were obtained following clinical practice guidelines and conserved as FFPE for further RNA extraction. PAM50 was performed on both digital multiplexed gene expression and RNA-Seq platforms. Subtype assignment was based on the nearest centroid classification following this procedure for both platforms and it was concordant on $96 \%$ of the cases $(N=96)$. In four cases, digital multiplexed gene expression analysis and RNA-Seq were discordant. The Spearman correlation to each of the centroids and the risk of recurrence were above 0.89 in both platforms while the agreement on Proliferation Score reached up to 0.97 . In addition, 82\% of the individual PAM50 genes showed a correlation coefficient $>0.80$.

Conclusions: In our analysis, the subtype calling in most of the samples was concordant in both platforms and the potential discordances had reduced clinical implications in terms of prognosis. If speed and cost are the main driving forces then the preferred technique is the digital multiplexed platform, while if whole genome patterns and subtype are the driving forces, then RNA-Seq is the preferred method.
\end{abstract}

Keywords: PAM50, Breast cancer, Triple negative breast cancer, RNA-Seq, Multiplexed gene expression

\footnotetext{
* Correspondence: antonio.picornell@iisgm.com

Presented in: ESMO 2017 Meeting (Madrid, Spain. 08 Sep - 12 Sep 2017)

'Instituto de Investigación Sanitaria Gregorio Marañón (IISGM), Doctor

Esquerdo 46, 28007 Madrid, Spain

Full list of author information is available at the end of the article
}

(c) The Author(s). 2019 Open Access This article is distributed under the terms of the Creative Commons Attribution 4.0 International License (http://creativecommons.org/licenses/by/4.0/), which permits unrestricted use, distribution, and reproduction in any medium, provided you give appropriate credit to the original author(s) and the source, provide a link to the Creative Commons license, and indicate if changes were made. The Creative Commons Public Domain Dedication waiver (http://creativecommons.org/publicdomain/zero/1.0/) applies to the data made available in this article, unless otherwise stated. 


\section{Background}

Gene expression signatures are becoming a key tool for decision-making in oncology, and especially in breast cancer. In 2000, Perou et al. identified 4 intrinsic subtypes of breast cancer with clinical implications from microarray gene expression data: Luminal A (LumA), Luminal B (LumB), HER2-enriched and Basal-like [1-3]. These breast cancer subtypes yielded a superior prognostic impact than classical immunohistochemistry (IHC) factors. Almost a decade later Parker et al. developed from the initial intrinsic subtypes, a 50-gene signature for subtype assignment [4].

Initially developed on microarray data, PAM50 is being successfully used in digital multiplexed gene expression platforms such as NanoString nCounter ${ }^{\oplus}$, which is the basis for the Prosigna ${ }^{\oplus}$ test. The latter includes the PAM50 assay in combination with a clinical factor (i.e. tumor size) and has been approved for the risk of distant relapse estimation in postmenopausal women with hormone receptorpositive, node negative or node positive early stage breast cancer patients; and is a daily-used tool assessing the indication of adjuvant chemotherapy [5, 6].

The NanoString nCounter ${ }^{\circledR}$ system enables gene expression analysis through direct multiplexed measurements. This technology is based on 2 probes specific to each gene of interest, a capture probe and a reporter probe, consisting of a complementary sequence to the target messenger RNA (mRNA) coupled to a color-coded tag [7]. Unique pairs of capture and reporter probes are designed for each gene of interest, and up to 800 genes can be analyzed simultaneously for a single sample. Tumor RNA and probes are hybridized together and following purification and alignment, they are identified and quantified by the analyzer. NanoString has proved to be highly reproducible, and has shown a high concordance between freshfrozen (FF) and formalin-fixed paraffin-embedded (FFPE) derived RNAs [8].

On the other hand, RNA-Seq has become the cornerstone of modern whole transcriptome analyses. It represents a useful tool for discovery and validation of biomarkers. The use of FFPE has been a concern in the past but several studies observed that this kind of samples are suitable to be used in RNA-Seq platforms assessing for gene expression analyses, and comparable to fresh frozen tissue [9]. From the technical point of view, typical RNASeq protocols based on poly(A) enrichment of the mRNA in order to remove ribosomal RNA, fail to capture the partially degraded mRNA in FFPE samples. However this limitation can be overcome by using Ribo-Zero-Seq and it has been proved that it performs as good as microarrays or RNA-Seq based on poly(A) enrichment [10]. However, its processing time requirements and economic costs make it difficult its implementation in daily clinical practice scenario. In this study, we compared the performance of the intrinsic subtype determination by PAM50 along with the risk of recurrence (ROR) estimation from both platforms: RNA-Seq and NanoString nCounter ${ }^{\odot}$, by using the same samples on both and directly comparing results.

\section{Results \\ Sample quality}

Overall, 96 samples were successfully processed and had sufficient RNA for both NanoString nCounter ${ }^{\circledR}$ and RNA-Seq transcript quantification. The mean RNA concentration from the FFPE samples was $146.9 \mathrm{ng} / \mu \mathrm{l}$, mean RNA integrity number (RIN) value was 2.015 ( $\mathrm{min} / \mathrm{max}$ : 1.1/3.7; 95\% CI: 1.899-2.130) and its mean A260/A280 ratio was 1.98 ( $\mathrm{min} / \max : 1.83 / 2.06$; 95\% CI: $1.971-$ 1.979) (Additional file 2: Table S2, online only). None of the samples used in RNA-Seq had measurable amounts of rRNA and all the samples presented optimal metrics. Moreover, the none of the samples processed in NanoString nCounter ${ }^{\ominus}$ presented technical issues and just three of them presented negligible control/count hints. Both quality control (QC) reports are in the respective Additional files 4 and 5 (online only).

\section{Intrinsic subtype calling}

The intrinsic subtype calling results in both RNA-Seq and NanoString nCounter ${ }^{\circ}$ are shown in the Additional file 1: Table S1 (online only).

As displayed in Fig. 1, NanoString nCounter ${ }^{\bullet}$ classified $84.3 \%$ of the patients as Basal-like, $11.5 \%$ as HER2enriched, 3.1\% as LumA and 1.0\% as LumB. RNA-Seq intrinsic subtype distribution was as follows: $78.1 \%$ basallike, 16.7\% HER2-enriched, 4.2\% LumA, 1.0\% LumB.

As displayed in Table 1, we had 7 patients with discordant subtype calls by the two techniques (7.3\%). However, we observed that 3 patients had their second closest centroids within a distance $\leq 0.10$ (range: 0.01 to 0.10 ), one of them concordant with the call offered by the other technique. The remaining 4 discordant cases showed real discordances in their calls and centroids proximity. Taking this information into account, we considered that subtype calling agreed on $96 \%$ of the cases (NanoString nCounter $/$ RNA-Seq discordances: 3 Basallike/HER2-enriched and 1 HER2-enriched/LumA). We reevaluated the discordant samples in the PAM50 assay output. We only observed that one sample (HUGM0022) had a low confidence score (0.42) in RNA-Seq due to extremely similar centroid correlation values, thus we really cannot classify it with a high degree of confidence.

\section{PAM50 centroids and risk of recurrence}

We next analyzed the correlation to each of the centroids obtained through NanoString nCounter ${ }^{\circledR}$ and RNA-Seq data, and we observed that the Spearman's rho was above 0.95 for all the centroids (Basal-like/HER2-enriched 0.97, LumA 0.95, and LumB 0.96) (Fig. 2). 
TNBC PAM50 calling
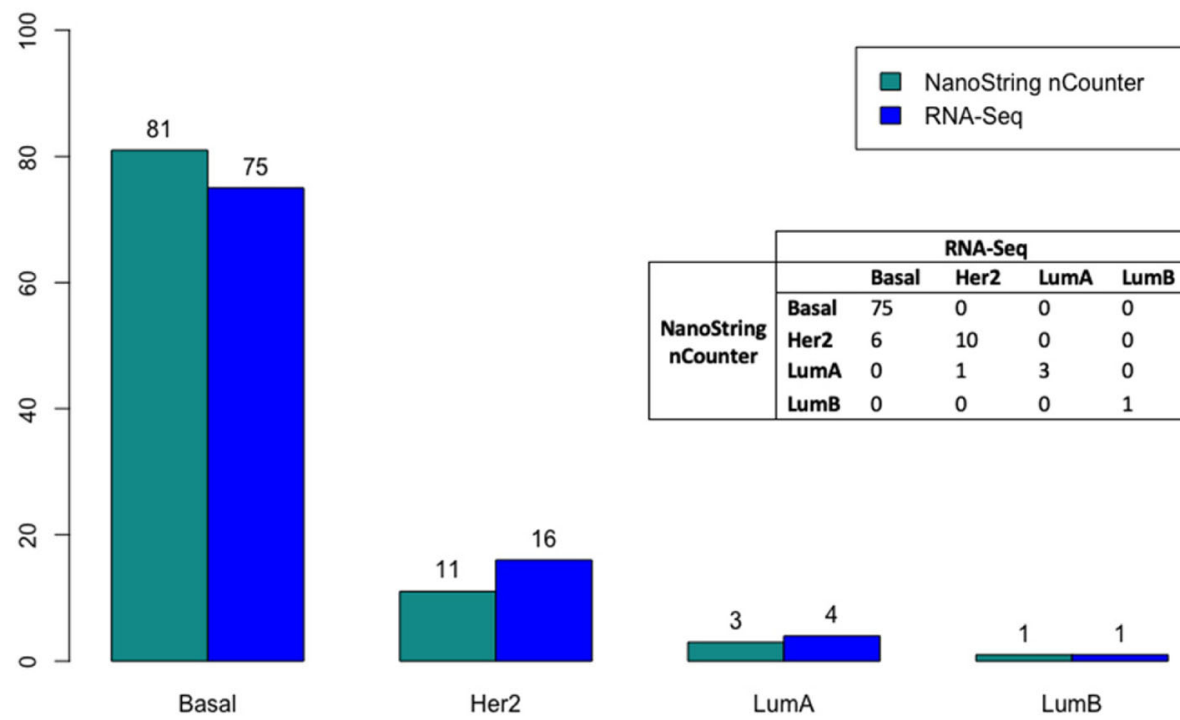

Fig. 1 PAM50 subtype calls by technique. Barplot represents counts of samples per subtype and technique. The cross table shows in detail the discordances between both platforms

In addition, we evaluated the correlation between each of the different centroids for both platforms and we observed similar results. The highest positive correlation was for the HER2-enriched and LumB centroids, with a Spearman's rho of 0.83 and 0.85 $(p<0.01)$ with RNA-Seq and NanoString nCounter ${ }^{\circ}$ respectively. On the other hand, Basal-like and LumA centroids had the strongest inverse correlation (rho 0.86 and $0.76, p<0.01$ with RNA-Seq and NanoString nCounter ${ }^{\circ}$, respectively) (Fig. 3).

Table 1 Centroid correlation for the potential discordant sample calls

\begin{tabular}{|c|c|c|c|c|c|c|}
\hline & Sample ID & Basal-like & HER2-enriched & Lum A & Lum B & Discordance \\
\hline \multirow{7}{*}{$\begin{array}{c}\text { NanoString } \\
\text { nCounter }\end{array}$} & HCSC-0027 & 0.44 & 0.08 & -0.28 & -0.29 & YES \\
\hline & HLPR-002 & 0.03 & 0.26 & -0.02 & -0.15 & YES \\
\hline & HUGM-0022 & 0.37 & 0.00 & -0.30 & -0.23 & YES \\
\hline & HUGM-0029 & 0.59 & 0.29 & -0.64 & -0.08 & NO (Basal) \\
\hline & HUGM-0047 & 0.41 & $0.40 *$ & -0.71 & 0.18 & NO (Her2) \\
\hline & INEN-0017 & 0.51 & 0.25 & -0.55 & -0.20 & NO (Basal) \\
\hline & INEN-0021 & 0.42 & 0.29 & -0.57 & 0.09 & YES \\
\hline \multirow{7}{*}{ RNA-Seq } & HCSC-0027 & -0.17 & 0.26 & $0.18^{*}$ & -0.08 & YES \\
\hline & HLPR-002 & -0.47 & 0.07 & 0.54 & -0.04 & YES \\
\hline & HUGM-0022 & 0.00 & 0.08 & $0.02 *$ & -0.05 & YES \\
\hline & HUGM-0029 & $0.39 *$ & 0.47 & -0.56 & 0.11 & NO (Basal) \\
\hline & HUGM-0047 & 0.07 & 0.51 & -0.46 & 0.34 & NO (Her2) \\
\hline & INEN-0017 & $0.26^{*}$ & 0.36 & -0.38 & -0.03 & NO (Basal) \\
\hline & INEN-0021 & 0.17 & 0.36 & -0.36 & 0.29 & YES \\
\hline
\end{tabular}

These measures are extracted from the PAM50 assay outcome (Additional file 1: Table S1). The sample's subtype classification is assigned to the centroid with the highest correlation (in bold red). When the second centroid has a value close to the highest one (difference less or equal to 0.1 ) the classification is ambiguous being possible any of both subtypes (bold *). The Discordance column summarizes whether a real discordance is observed in a sample or just a scenario where two centroid correlations are almost equivalent (HUGM-0047 in NanoString nCounter ${ }^{\circledast}$ is a paradigmatic case) 


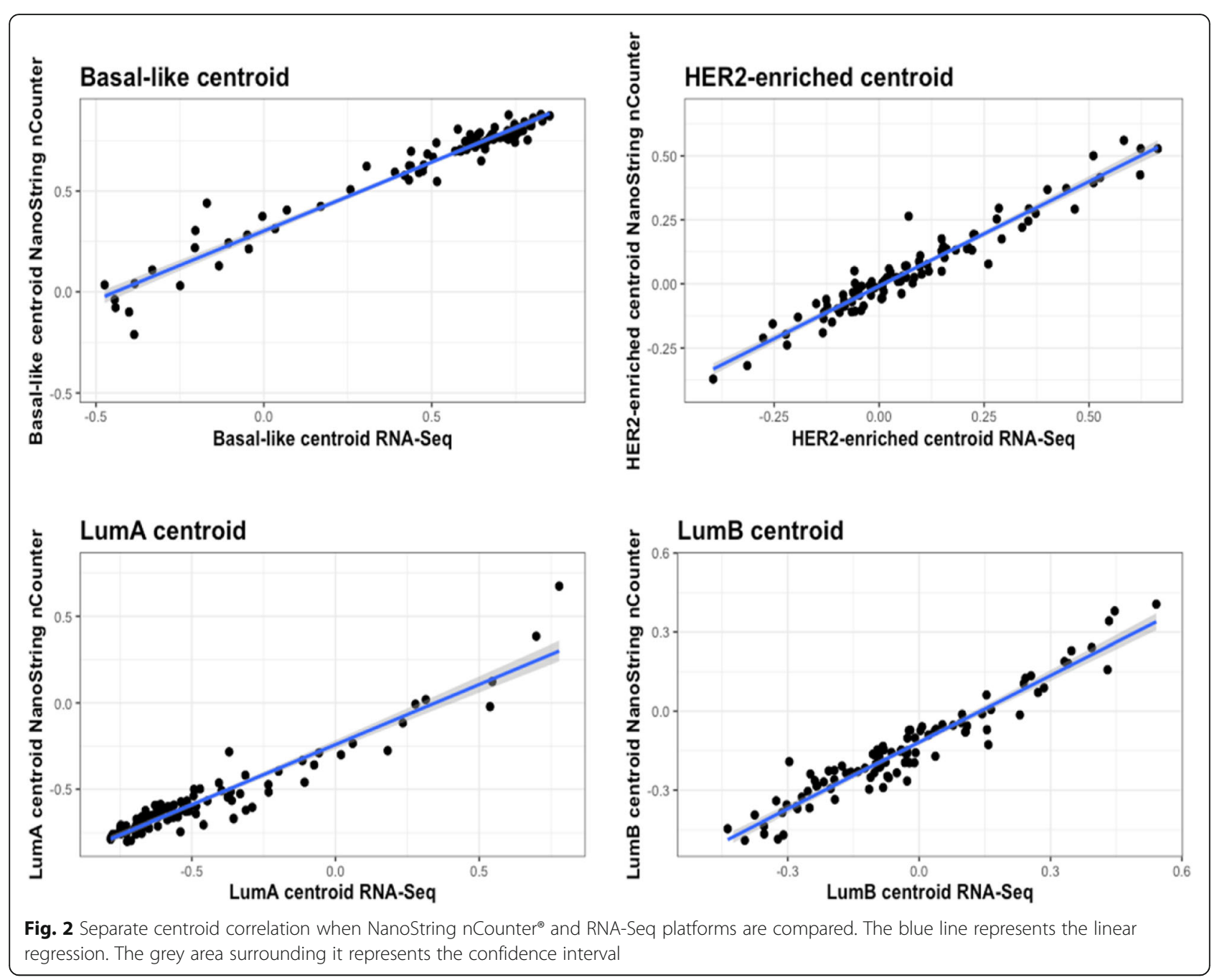

The risk of recurrence score (ROR), and considering the role of the Proliferation Score (ROR + PS), had a Spearman's rho of 0.90 and 0.97 , respectively. Thus, in terms of ROR, the results show an extremely high correlated scenario. We observed high agreement between techniques in the Bland-Altman plots displayed in Fig. 4, as most of the differences remain close to the null baseline level within the confidence interval. In addition, the intraclass correlation coefficient (ICC) for ROR reached 0.93 [0.89-0.95] and ROR + PS reached 0.96 [0.94-0.97].

Additional measures such as expression level of HER2, along with the Proliferation Score, also showed a high degree of correlation between both platforms with a Spearman's rho 0.96 and 0.97 , respectively.

\section{Individual gene correlation}

We lastly evaluated the correlation coefficients for each of the 50 genes in the PAM50 gene list. We measured the expression levels in $\log 2$ scale in both platforms. We observed that in our dataset 23 genes had a correlation greater than $0.9,18$ genes between 0.8 and $0.9,7$ genes between 0.7 and 0.8 and only 2 genes had a correlation lower than 0.7. The median ICC was $0.90($ mean $=0.88)$ (Fig. 5 and Additional file 3: Table S3, online only).

\section{Discussion}

The goal of the study was assessing the reproducibility of PAM50 intrinsic subtype when using RNA-Seq and NanoString nCounter ${ }^{\circ}$ data from FFPE tissue obtained from a triple negative breast cancer (TNBC) patient cohort. We noticed that the PAM50 subtype calling was concordant on $96 \%$ of the cases and the expression in genes that comprise the PAM50 assay had a median ICC of 0.90 .

PAM50 was originally developed and validated using microarray data from 1753 genes, but since then it has been transferred into a wide variety of platforms. Interestingly, PAM50 performance has been evaluated by comparing quantitative real-time reverse-transcriptionPCR (qRT-PCR) and NanoString nCounter ${ }^{\circ}$ [11]. That study obtained an overall concordance of 0.94 in subtype 
PAM50 RNA-Seq centroids
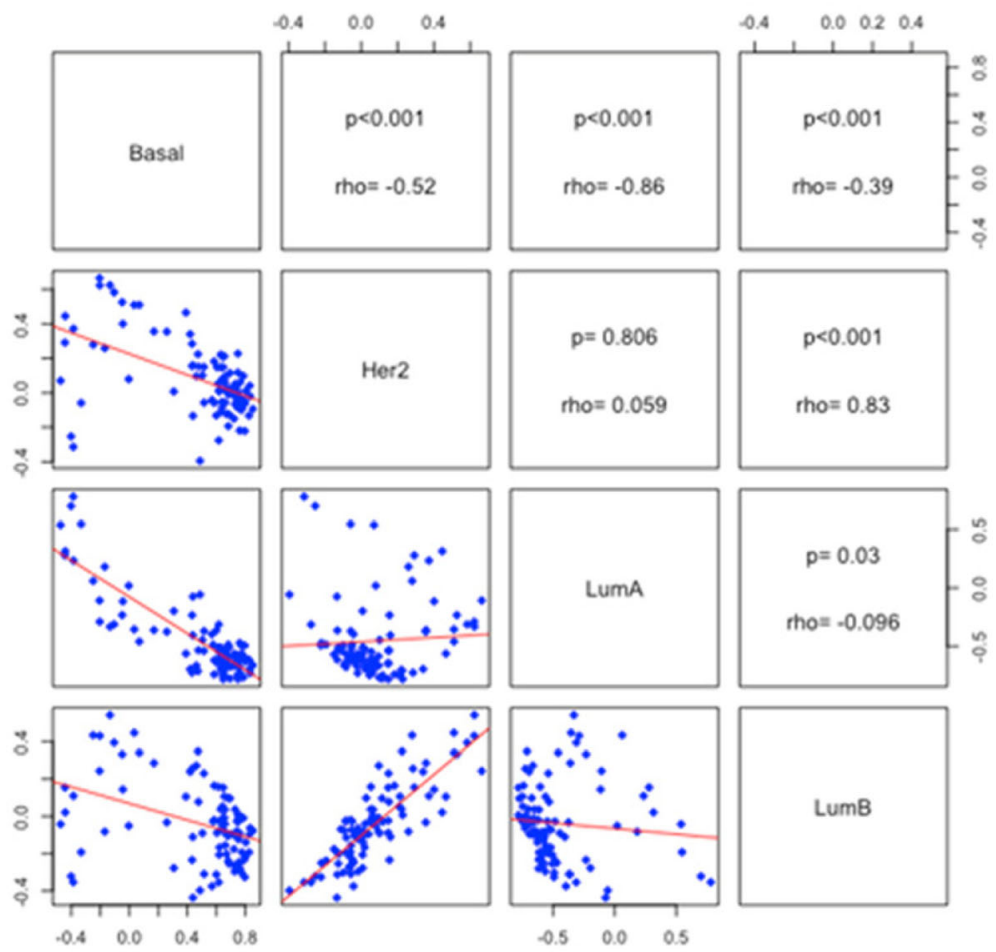

PAM50 NanoString nCounter centroids
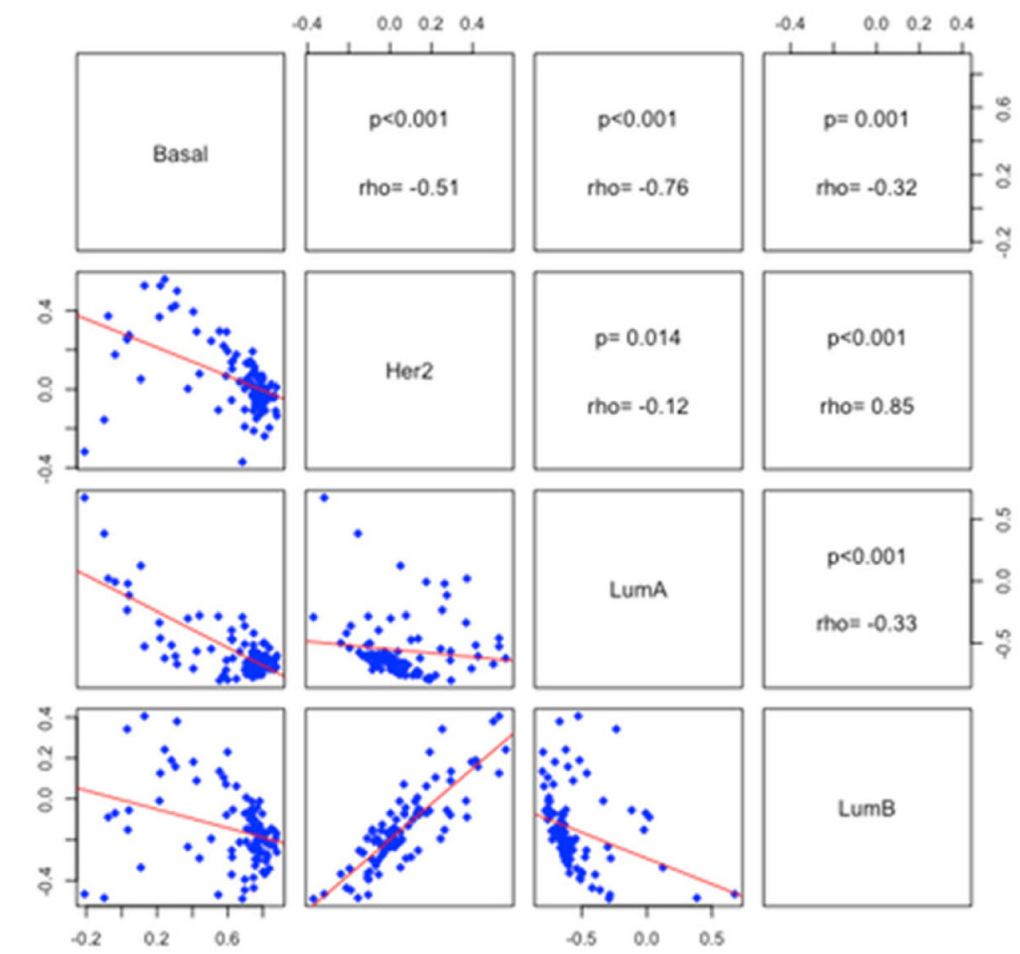

Fig. 3 Correlation of the correlation to the centroids in both platforms obtained in the PAM50 subtype classifier 

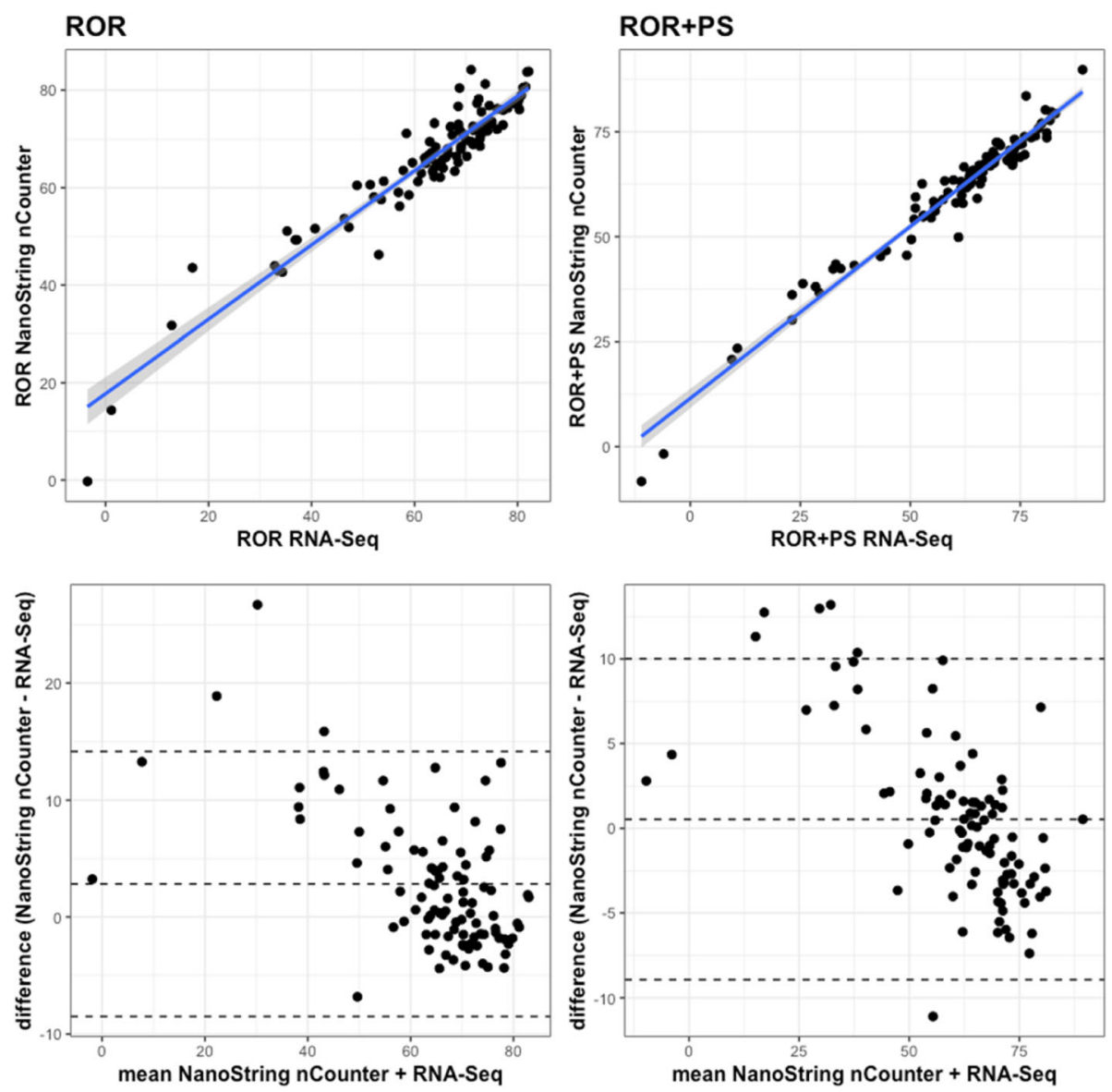

Fig. 4 Correlation of ROR and ROR +PS and their associated Bland-Altman plots in both platforms. The upper/lower dashed lines in the BlandAltman plots represent the mean difference $+/-1.96 *$ standard deviation. The central dashed line represents the mean difference

calls, 0.98 for ROR and 0.95 for ROR + PS. Regarding individual gene expression, median ICC was 0.90 [11]. These measures are very similar to ours comparing NanoString nCounter ${ }^{\circ}$ and RNA-Seq, as we presented in the Results Section.

In this TNBC cohort 4 samples out of 96 were misclassified in the subtype calling. While this might be concerning from the patient care perspective, it is strongly suggested in these cases to evaluate the ROR and ROR + PS, because from the clinical point of view the ROR-score group is more important to select therapy (chemotherapy vs no chemotherapy) than the plain subtype calling. The PAM50 assay provides numeric and categorical values for both scores and we observed in the misclassified samples the assigned risk group remained the same except in one patient with discordant low/medium ROR (Table 2).

Perou, Sørlie, Hu, Nielsen et al. evaluated the prognostic effect of PAM50 genes using the qRT-PCR from FFPE samples, and demonstrated its superiority to standard clinicopathological factors in predicting long-term survival of estrogen receptor positive tumors $[12,13]$. There is significant evidence that IHC is not a reliable surrogate of genomic intrinsic subtype, and that gene expression methods have a higher predictive and prognostic value than IHC [12, 14, 15]. Moreover, in a comprehensive review in breast cancer gene-expression based assays by Prat et al. it is shown that the concordance between two different ER/ PR testing methods based on IHC falls below the highest levels of reproducibility/concordance expected in daily clinical use [16].

The kind of samples to be processed is often a major factor in deciding which technology should be used to quantify transcripts and perform the PAM50 assay. In medical research the FFPE are the most common sources of archived material because they are cheap, easy to process and stable for a very long time. The PAM50 PCRbased classifier has been validated and translated into the NanoString nCounter ${ }^{\circ}$ platform, because it previously demonstrated higher performance than PCR for FFPE data [8]. Since this platform does not require an amplification step, it enables a more sensitive analysis of degraded mRNA from FFPE samples [17, 18]. Although it seems that NanoString and DNA microarrays show a good correlation, similar to the one found when comparing distinct 

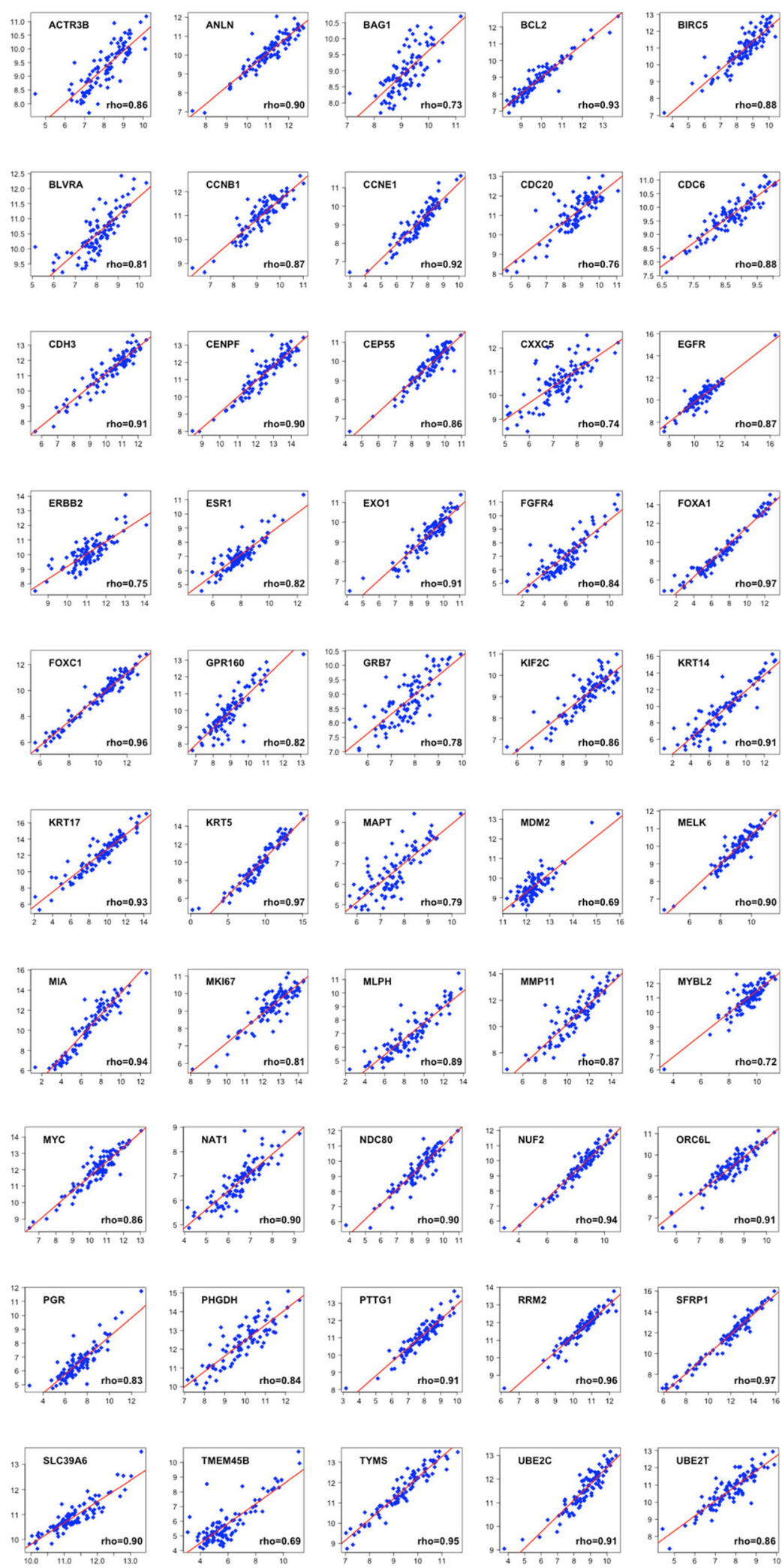

Fig. 5 Normalized gene expression levels for each gene contained in the PAM50 assay. The log2 normalized counts for RNA-Seq are represented in the $\mathrm{X}$-axis and those for NanoString nCounter ${ }^{\bullet}$ are represented in the $\mathrm{Y}$-axis. The red line represents the LOWESS smoother, which uses locally weighted polynomial regression 
Table 2 Risk of Recurrence (ROR) for the potential discordant sample calls

\begin{tabular}{|c|c|c|c|c|c|c|}
\hline & Sample ID & $\mathrm{ROR}$ & ROR-Group & ROR-PS & ROR + PS-Group & Discordance \\
\hline \multirow[t]{7}{*}{ NanoString nCounter } & HCSC-0027 & 51.10 & med & 43.42 & med & $\mathrm{NO}$ \\
\hline & HLPR-002 & 43.57 & med & 23.44 & med & YES \\
\hline & HUGM-0022 & 51.60 & med & 42.28 & med & NO \\
\hline & HUGM-0029 & 73.51 & high & 68.98 & high & NO \\
\hline & HUGM-0047 & 81.24 & high & 72.48 & high & NO \\
\hline & INEN-0017 & 66.66 & high & 55.051 & high & NO \\
\hline & INEN-0021 & 72.50 & high & 65.40 & high & NO \\
\hline \multirow[t]{7}{*}{ RNA-Seq } & HCSC-0027 & 35.25 & med & 33.04 & med & NO \\
\hline & HLPR-002 & 16.87 & low & 10.68 & low & YES \\
\hline & HUGM-0022 & 40.68 & med & 32.44 & med & NO \\
\hline & HUGM-0029 & 75.02 & high & 69.59 & high & NO \\
\hline & HUGM-0047 & 73.73 & high & 69.58 & high & NO \\
\hline & INEN-0017 & 62.68 & high & 52.98 & high & NO \\
\hline & INEN-0021 & 66.98 & high & 64.52 & high & NO \\
\hline
\end{tabular}

These measures are extracted from the PAM50 assay outcome (Additional file 1: Table S1). Two measures regarding the risk of recurrence are reported: ROR, which takes into account only the subtype calling; and ROR + PS, which considers also the Proliferation Score. The latter is defined as the mean expression level for the proliferation genes: CCNB1, UBE2C, BIRC5, KNTC2, CDC20, PTTG1, RRM2, MK167, TYMS, CEP55 and CDCA1. The ROR(+PS)-Group columns gives a categorical classification in terms of risk: high, medium (med) and low

microarrays platforms [7], correlations between FF microarrays and FFPE was moderate due to RNA poor quality in the FFPE samples. According to Chen et al. NanoString detected a higher number of transcripts than microarrays (88.4\% vs. $82.6 \%$ ) [17].

Several studies for both oncologic and non-oncologic diseases have shown a good correlation between gene expression data from RNA-Seq and NanoString nCoun$\operatorname{ter}^{\circledast}\left(R^{2}=0.90\right.$ for FFPE samples in idiopathic pulmonary fibrosis) [19]. Particularly in breast cancer, NanoString nCounter ${ }^{\bullet}$ and RNA-Seq using Illumina TruSeq RiboZero-Gold RNA-Seq enable reliable gene expression analysis from degraded FFPE RNA. This study encourages the role of NanoString nCounter ${ }^{\bullet}$ as a validation platform from data discovered by RNA-Seq, with a high reproducibility of both techniques $\left(R^{2}=0.99\right.$ for technical replicates), high correlation with FF matched samples $\left(R^{2}=0.874\right.$ for NanoString) and a high correlation between both platforms $\left(R^{2}=0.838\right)$ [8].

While RNA-Seq technologies can be used in multiple research scenarios [20] a digital multiplexed platform provides several advantages over RNA-Seq technologies in daily clinical practice in terms of cost, amount of needed RNA, computational cost and the use of FFPE samples. This last factor is often crucial in clinical research as fresh frozen (FF) tissue is generally unavailable due to the infrastructure and timing required, making it difficult to reach the necessary number of samples in multicenter studies. However, that means increased sequencing costs in order to get enough reads to achieve adequate coverage of coding genes. In this project the estimated full cost for processing and sequencing an RNA-Seq sample was around 800-1000 USD; on the other hand, the cost of processing a sample in NanoString nCounter ${ }^{\circ}$ was just around 190 USD. The overall financial burden to perform PAM50 using NanoString nCounter ${ }^{\circ}$ in a diagnostic laboratory is lower than any RNA-Seq option in terms of equipment and experts, as the latter may require outsourcing the analyses. This situation usually entails longer delivery times as long as the sample processing and transcript quantification using NanoString nCounter ${ }^{\oplus}$ can be achieved in less than $28 \mathrm{~h}$. In addition, the amount of RNA needed in both platforms is substantially different: in our case we ran the analyses using 500$1000 \mathrm{ng}$ in RNA-Seq and only $250 \mathrm{ng}$ in NanoString nCounter; the latter claims to keep its performance with only $125 \mathrm{ng}$. Additionally, the computational costs can be several orders of magnitude higher in processing RNA-Seq than NanoString nCounter ${ }^{\circledast}$ samples because the former needs computationally demanding processes of alignment and transcript quantification.

Finally, our study shows that PAM50 intrinsic subtype in TNBC patients, which are mostly basal-like, is reproducible between different platforms. This encourages the solidity of the classification. Moreover, our comparison comes from the same FFPE-extracted RNA, directly comparing the platform with no RNA quality bias.

\section{Conclusions}

The PAM50 subtype calling agreement between RNA-Seq and NanoString nCounter ${ }^{\bullet}$ transcript quantification technologies was evaluated in FFPE tissue samples obtained from a TNBC patient cohort. We observed that the subtype 
call was concordant in most of the samples and the potential discordances had reduced clinical implications in terms of prognosis. Although PAM50 can be used with RNA-Seq data and it shows similar results to NanoString nCounter; the former is still difficult to use in daily clinical practice due to its processing time requirements and economic costs, unlike the discussed digital multiplexed option.

\section{Methods}

\section{Patients and samples}

The analyses were performed in FFPE samples from 96 patients recruited in a previously described multicenter, prospective, non-randomized neoadjuvant TNBC trial (NCT01560663) [21]. This cohort consisted of early stage TNBC patients, defined as estrogen and progesterone receptor (ER and PR) $<1 \%$ and HER2-negative according to the ASCO-CAP guidelines, candidate for neoadjuvant treatment with carboplatin and docetaxel $[22,23]$.

This trial included patients from 7 centers across Spain and Peru. Pre-treatment core biopsies were performed and conserved as FFPE following each institution protocol for further RNA extraction.

This study was reviewed and approved by the Ethical Board at all the participating institutions, and all patients included were required to sign a written informed consent. All clinical data and samples were anonymized.

\section{RNA extraction}

FFPE blocks were centralized and included in a biologic sample bank at the Translational Oncology Laboratory (LAOT), which belongs to the Medical Oncology Department at the Hospital General Universitario Gregorio Marañón, registered at the Instituto de Salud Carlos III; in Madrid, Spain.

RNA extraction and PAM50 intrinsic subtyping on the nCounter ${ }^{\circledast}$ platform were performed at the LAOT. Invasive tumor from haematoxilin and eosin stained slides was delimited by a pathologist at the LAOT and was subsequently microdissected in $10 \mu \mathrm{m}$ slides. The number of slides recommended based on the measured tumor surface area on the hematoxylin and eosin (H\&E) stained slide; $4-19 \mathrm{~mm}^{2}$ : 6 unstained $10 \mu \mathrm{m}$ slides; 20$99 \mathrm{~mm}^{2}$ : 3 unstained $10 \mu \mathrm{m}$ slides; $\geq 100 \mathrm{~mm}^{2}$ : 1 unstained $10 \mu \mathrm{m}$ slide. RNA was then extracted using the RNeasy FFPE Kit (Qiagen), which is specially designed for purification of total RNA from FFPE tissue sections by isolating RNA molecules longer than 70 nucleotides. Firstly, all paraffin is removed from freshly cut FFPE tissue sections by treating with deparaffinization solution or using an alternative deparaffinization method. Next, samples are incubated in an optimized lysis buffer, which contains proteinase $K$, to release RNA from the sections. A short incubation at a higher temperature partially reverses formalin crosslinking of the released nucleic acids, improving RNA yield and quality, as well as RNA performance in downstream enzymatic assays. This is followed by DNase treatment that is optimized to eliminate all genomic DNA, including very small DNA fragments that are often present in FFPE samples after prolonged formalin fixation and/or long storage times. Next, the lysate is mixed with Buffer RBC. Ethanol is added to provide appropriate binding conditions for RNA, and the sample is then applied to a RNeasy MinElute spin column, where the total RNA binds to the membrane and contaminants are efficiently washed away. RNA is then eluted in a minimum of $14 \mu \mathrm{l}$ of RNase-free water. RNA concentration and quality were assessed with Nanodrop 2000 Spectophotometer (Thermo Scientific NanoDrop Products) according to A260/280 ratio. RIN was evaluated with the TapeStation 2200 (Agilent Technologies, Germany)

\section{NanoString nCounter ${ }^{\circledast}$}

From an initial panel of 110 genes, we analyzed the expression of the 50 genes included in the PAM50 assay and 5 additional housekeeper genes described by Parker et al [4]. In the raw nCounter ${ }^{\bullet}$ transcript quantification the background was corrected using the negative probes and normalized with their mean minus 2 standard deviations, and those values were normalized by calculating the geometric mean of the 5 housekeeper genes. Subtype classification was assigned based on the nearest of the 5 centroids.

\section{RNA-Seq}

Sequencing was performed at the University of North Carolina (UNC) at Chapel Hill (NC, US). 300-1000 ng of total FFPE RNA was used to create an RNA-Seq library using the Illumina TruSeq Ribo-Zero Gold Kit (RS - 1 22-2301 or RS - 122 - 2302). Libraries were then sequenced 2 per lane on a HiSeq 2500 with $48 \times 7 \times 48$ bp configuration. Alignment against GRCh37 and transcript quantification was done using MapSplice [23] v2.2.1 and RSEM [4] v1.3.0 using the UCSC GAF2.1 KnownGenes using UBU v1.0 (https://github.com/mozack/ubu), respectively. Samples were normalized to a fixed upper quartile (1000 genes) followed by $\log 2$ transformation. The PAM50 algorithm was run according to the scripts provided by Parker et al [4] adjusting the genes to a previously determined estrogen receptor balanced median from FFPE samples assayed by TruSeq Ribo-Zero Gold to adjust samples to match the training set. The code and median adjustment value are provided as Additional file 6 .

\section{Quality control analysis}

The quality control for the RNA-Seq samples was assessed using the fastq files in FastQC v0.11.8 [24]. We also 
evaluated the potential presence of ribosomal RNA sequences obtained from the UCSC Genome Browser [25] using FastQ Screen v0.13.0 [26]. The QC for the NanoString nCounter samples was done using NanoStringQCPro [27].

\section{Statistical analysis}

We used R [28] v3.3.3 in order to evaluate the transcript quantification in both platforms, and the PAM50 subtype calling along with its associated centroid values and the ROR. All comparisons between continuous variables were performed using the Spearman correlation. The inter-rater agreement analysis for molecular subtype classification was based on the Cohen's kappa calculation. For continuous variables ICC two way mixed effect single measures [29] was calculated. Given ROR importance in diagnostic, a Bland-Altman plot was constructed to further assess agreement between platforms.

\section{Additional files}

Additional file 1: Table S1. PAM50 output for RNA-Seq and NanoString nCounter. PAM50 table of results for RNA-Seq and NanoString nCounter ${ }^{\circ}$. It is reported for each sample and platform: the centroid correlation, the subtype call with its confidence, the ROR and ROR + PS values and groups, the Proliferation Score; and the ER/HER2 gene expression. (XLSX $90 \mathrm{~kb})$

Additional file 2: Table S2. RNA Sample Quality Control. The RNA concentration measured in each sample, absorbance values for A260 and A280, the A260/A280 ratio and the measured RIN. Descriptive statistics are provided for A260/A280 ratio and RIN. (XLSX $16 \mathrm{~kb}$ )

Additional file 3: Table S3. PAM50 genes correlation and inter-rate agreement analyses. Correlation analysis using the Spearman's rho where the gene expression measured in RNA-Seq and NanoString nCounter ${ }^{\circledast}$ are compared. The inter-rater agreement analysis for the PAM50 genes was based on the Cohen's kappa calculation using two-way mixed effect single measures. The statistics and $p$-values are provided in both analyses. (XLSX $17 \mathrm{~kb}$ )

Additional file 4: QC - Fastq. RNA-Seq data Quality Control. FastQC and FastQ Screen reports summarized using MultiQC. All the information is summarized in an interactive .html. Additionally, a table is provided to associate the sample IDs mentioned in the manuscript with the IDs generated during the sequencing process. (ZIP $5962 \mathrm{~kb}$ )

Additional file 5: QC - NanoString. NanoString nCounter data Quality Control. NanoStringQCPro reports in .html files. Technical, control and count-based metrics are reported. Additionally, a table is provided to associate the sample IDs mentioned in the manuscript with the IDs generated during the NanoString nCounter ${ }^{\circledast}$ quantification process. (ZIP 15743 $\mathrm{kb})$

Additional file 6: Code. Normalization and PAM50 scripts for both platforms. Scripts used to perform the PAM50 assay in RNA-Seq and NanoString nCounter ${ }^{\oplus}$ platforms, along with additional technique-specific and transcript ID files. (ZIP $91 \mathrm{~kb})$

\section{Abbreviations}

ER: Estrogen receptor; FF: Fresh-frozen; FFPE: Formalin-fixed paraffinembedded; FISH: Fluorescence in situ hybridization; HER2: Human epidermal growth factor receptor 2; ICC: Intraclass correlation coefficient; IHC: Immunohistochemistry; mRNA: messenger RNA; PAM50: Prediction analysis for microarrays (50); PCR: Polymerase chain reaction; PR: Progesterone receptor; PS: Proliferation Score; QC: Quality control; qRTPCR: Real-Time Quantitative Reverse Transcription PCR; RIN: RNA integrity number; RNA: Ribonucleic acid; RNA-Seq: RNA sequencing; ROR: Risk of recurrence; TNBC: Triple negative breast cancer; UCSC: University of California, Santa Cruz

\section{Acknowledgements}

None.

\section{Authors' contributions}

All authors read and approved the final manuscript. APC: Wrote the final version of the manuscript; performed the main analyses in both platforms. IE: Conducted the PAM50 analyses in RNA-Seq; participated in the data managing process; manuscript writing. EA: Conducted basic statistical analyses; manuscript review. SLT: Patient recruitment; medical and manuscript advisoring and writing. YJ: Patient recruitment; medical and manuscript advisoring and writing. KH: Gathered and parsed the RNA-Seq results at the UNC and the first approach to PAM50 analyses; participated in the manuscript writing and reviews. JSP: Developed the PAM50 assay and advised about the way to run the analyses in both platforms; participated in the manuscript writing and reviews. MMM: Project and data manager; manuscript writing, editing and advisoring. RRM: Performed the RNA-seq extraction in the LAOT; participated in the technical dicussion and writing of methods in the manuscript. JG: Performed the RNA-seq extraction in the LAOT; participated in the technical dicsussion and writing of methods in the manuscript. IO: Performed the laboratory tasks using the NanoString nCounter platform; participated in the technical discussion of methods in the manuscript. MC: Medical (Anatomical Pathology) advisoring; pathological classification of the patients. TM: LAOT organization; patient recruitment; manuscript and medical review advisor. FM: Patient recruitment; manuscript and medical review advisor. JAGS: Patient recruitment; manuscript and medical review advisor. HGM: Patient recruitment; manuscript and medical review advisor. AB: Patient recruitment; manuscript and medical review advisor. MRB: Patient recruitment; manuscript and medical review advisor. CMP: Sample gathering and organization during the RNA-Seq process at UNC; manuscript writing and medical/technical review advisor. MM: Patient recruitment; manuscript writing and medical review advisor; project leader.

\section{Funding}

M.M was supported by two research grants from Ministry of Economy and Competitiveness ISCIII-FIS grants (PI 12/02684): "Predictores genómicos de respuesta a la quimioterapia neoadyuvante con docetaxel-carboplatino en pacientes con cáncer de mama triple negativo"/"Genomic predictors of response to neoadjuvant chemotherapy with docetaxel-carboplatin in patients with triple negative breast cancer"; and (PI 15/00117): "Cáncer de mama triple negative: Predicción de respuesta a docetaxel-carboplatino neoadyuvante mediante caracterización de TILs y firmas inmunes basadas en secuenciación masiva de RNA"/" Triple negative breast cancer: Prediction of response to neoadjuvant docetaxel-carboplatin by characterization of TILs and immune signatures based on massive RNA sequencing".

C.M.P was supported by funds from the NCI Breast SPORE program (P50CA58223)

The funding sources had no role in the design of this study, its execution, analyses, interpretation of the data, or decision to submit results.

\section{Availability of data and materials}

The datasets used and/or analysed during the current study are available from the corresponding author on reasonable request.

\section{Ethics approval and consent to participate}

The study was approved by the Spanish Health Authority (MMJ-CAR-201401) and by the ethics committees at all participating institutions: CEIC Area 7 Hospital Clínico San Carlos, CEIC Hospital de la Santa Cruz y San Pablo, CEIC del Hospital Universitari Vall d'Hebron, CEIC del Instituto Nacional de Enfermedades Neoplásicas "Dr. Eduardo Cáceres Grazini", CEIC GAE La Princesa Area 2, CEIC Area Valladolid Este and CEIC Area 1- Hospital General Universitario Gregorio Marañón at Instituto de Investigación Sanitaria Gregorio Marañón (liSGM) as reference Ethical Committee. The study was finally registered at clinicaltrials.gov (NCT01560663). All patients signed a written informed consent. 


\section{Consent for publication}

No individual person's data is provided neither was present in any step of the performed analyses.

\section{Competing interests}

C.M.P is an equity stock holder, consultant, and Board of Director Member, of BioClassifier LLC. C.M.P is also listed an inventor on patent applications on the Breast PAM50 assay. J.S.P is an inventor on patent applications on the Breast PAM50 assay.

\section{Publisher's Note}

Springer Nature remains neutral with regard to jurisdictional claims in published maps and institutional affiliations.

\begin{abstract}
Author details
${ }^{1}$ Instituto de Investigación Sanitaria Gregorio Marañón (liSGM), Doctor Esquerdo 46, 28007 Madrid, Spain. ${ }^{2}$ Hospital General Universitario Gregorio Marañón, Madrid, Spain. ${ }^{3}$ Medical Oncology Service, Instituto de Investigación Sanitaria Gregorio Marañón (liSGM). CiberOnc, Hospital General Universitario Gregorio Marañón, Madrid, Spain. ${ }^{4}$ Department of Genetics, University of North Carolina at Chapel Hill, Chapel Hill, NC, USA. ${ }^{5}$ Anatomical Pathology Service, Hospital General Universitario Gregorio Marañón, Madrid, Spain. ${ }^{6}$ Medical Oncology Service, Hospital Universitario Clínico San Carlos, Madrid, Spain. ${ }^{7}$ Medicina Oncológic, Instituto Nacional de Enfermedades Neoplásicas (INEN), Lima, Peru. ${ }^{8}$ Medical Oncology Service, Hospital Universitario de La Princesa, Madrid, Spain. ${ }^{9}$ Hospital Virgen del Rocío, Sevilla, Spain. ${ }^{10}$ Department of Genetics, Lineberger Comprehensive Cancer Center, University of North Carolina, Chapel Hill, NC, USA. ${ }^{11}$ Hospital General Universitario Gregorio Marañón, Instituto de Investigación Sanitaria Gregorio Marañón (liSGM), Universidad Complutense, CiberOnc, GEICAM, Madrid, Spain.
\end{abstract}

\section{Received: 28 September 2018 Accepted: 27 May 2019}

\section{Published online: 03 June 2019}

\section{References}

1. Perou CM, Sørlie T, Eisen MB, van de Rijn M, Jeffrey SS, Rees CA, et al. Molecular portraits of human breast tumours. Nature. 2000;406(6797): 747-52.

2. Sørlie T, Perou CM, Tibshirani R, Aas T, Geisler S, Johnsen H, et al. Gene expression patterns of breast carcinomas distinguish tumor subclasses with clinical implications. Proc Natl Acad Sci U S A. 2001;98(19):10869-74.

3. Hu Z, Fan C, Oh DS, Marron JS, He X, Qaqish BF, et al. The molecular portraits of breast tumors are conserved across microarray platforms. BMC Genomics. 2006;7(1):96.

4. Parker JS, Mullins M, Cheang MC, Leung S, Voduc D, Vickery T, et al. Supervised risk predictor of breast cancer based on intrinsic subtypes. J Clin Oncol. 2009:27(8):1160-7.

5. Gnant M, Filipits M, Greil R, Stoeger H, Rudas M, Bago-Horvath Z, et al. Predicting distant recurrence in receptor-positive breast cancer patients with limited clinicopathological risk: using the PAM50 risk of recurrence score in 1478 postmenopausal patients of the ABCSG-8 trial treated with adjuvant endocrine therapy alone. Ann Oncol. 2014;25(2):339-45.

6. Dowsett M, Sestak I, Lopez-Knowles E, Sidhu K, Dunbier AK, Cowens JW, et al. Comparison of PAM50 risk of recurrence score with Oncotype DX and IHC4 for predicting risk of distant recurrence after endocrine therapy. J Clin Oncol. 2013;31(22):2783-90.

7. Geiss GK, Bumgarner RE, Birditt B, Dahl T, Dowidar N, Dunaway DL, et al. Direct multiplexed measurement of gene expression with color-coded probe pairs. Nat Biotechnol. 2008;26(3):317-25.

8. Reis PP, Waldron L, Goswami RS, Xu W, Xuan Y, Perez-Ordonez B, et al. mRNA transcript quantification in archival samples using multiplexed, colorcoded probes. BMC Biotechnol. 2011;11(1):46.

9. Jovanović B, Sheng Q, Seitz RS, Lawrence KD, Morris SW, Thomas LR, et al. Comparison of triple-negative breast cancer molecular subtyping using RNA from matched fresh-frozen versus formalin-fixed paraffin-embedded tissue. BMC Cancer. 2017;17(1). [cited 2018 Jan 4] Available from: https:// bmccancer.biomedcentral.com/articles/10.1186/s12885-017-3237-1.

10. Zhao W, He X, Hoadley KA, Parker JS, Hayes DN, Perou CM. Comparison of RNA-Seq by poly (a) capture, ribosomal RNA depletion, and DNA microarray for expression profiling. BMC Genomics. 2014;15(1):1.
11. Wallden B, Storhoff J, Nielsen T, Dowidar N, Schaper C, Ferree S, et al. Development and verification of the PAM50-based Prosigna breast cancer gene signature assay. BMC Med Genomics. 2015;8(1). [cited 2016 Sep 5] Available from: https://www.biomedcentral.com/1755-8794/8/54

12. Nielsen TO, Parker JS, Leung S, Voduc D, Ebbert M, Vickery $T$, et al. A comparison of PAM50 intrinsic subtyping with immunohistochemistry and clinical prognostic factors in tamoxifen-treated estrogen receptor-positive breast Cancer. Clin Cancer Res. 2010;16(21):5222-32.

13. Bastien RRL, Rodríguez-Lescure Á, Ebbert MTW, Prat A, Munárriz B, Rowe L, et al. PAM50 breast cancer subtyping by RT-qPCR and concordance with standard clinical molecular markers. BMC Med Genet. 2012;5:44.

14. Tutt A, Ellis P, Kilburn L, Gilett C, Pinder S, Abraham J. TNT: a randomized phase III trial of carboplatin compared with docetaxel for patients with metastatic or recurrent locally advanced triple negative or BRCA 1/2 breast cancer. San Antonio; 2014.

15. Cheang MCU, Martin M, Nielsen TO, Prat A, Voduc D, Rodriguez-Lescure A, et al. Defining breast Cancer intrinsic subtypes by quantitative receptor expression. Oncologist. 2015;20(5):474-82.

16. Prat A, Ellis MJ, Perou CM. Practical implications of gene-expression-based assays for breast oncologists. Nat Rev Clin Oncol. 2012;9(1):48-57.

17. Chen X, Deane NG, Lewis KB, Li J, Zhu J, Washington MK, et al. Comparison of Nanostring nCounter ${ }^{\oplus}$ data on FFPE Colon Cancer samples and Affymetrix microarray data on matched frozen tissues. Wang $X$, editor. PLoS One. 2016;11(5):e0153784.

18. Veldman-Jones MH, Lai Z, Wappett M, Harbron CG, Barrett JC, Harrington EA, et al. Reproducible, quantitative, and flexible molecular subtyping of clinical DLBCL samples using the NanoString nCounter system. Clin Cancer Res. 2015;21(10):2367-78.

19. Vukmirovic M, Herazo-Maya JD, Blackmon J, Skodric-Trifunovic V, Jovanovic D, Pavlovic S, et al. Identification and validation of differentially expressed transcripts by RNA-sequencing of formalin-fixed, paraffin-embedded (FFPE) lung tissue from patients with Idiopathic Pulmonary Fibrosis. BMC Pulm Med. 2017;17(1):15.

20. Wang Z, Gerstein M, Snyder M. RNA-Seq: a revolutionary tool for transcriptomics. Nat Rev Genet. 2009;10(1):57-63.

21. Sharma P, López-Tarruella S, García-Saenz JA, Ward C, Connor CS, Gómez $\mathrm{HL}$, et al. Efficacy of neoadjuvant carboplatin plus docetaxel in triplenegative breast Cancer: combined analysis of two cohorts. Clin Cancer Res. 2017;23(3):649-57.

22. Hammond MEH, Hayes DF, Dowsett M, Allred DC, Hagerty KL, Badve S, et al American Society of Clinical Oncology/College of American Pathologists Guideline Recommendations for Immunohistochemical testing of estrogen and progesterone receptors in breast Cancer. J Clin Oncol. 2010;28(16): 2784-95.

23. Wolff AC, Hammond MEH, Hicks DG, Dowsett M, McShane LM, Allison KH, et al. Recommendations for human epidermal growth factor receptor 2 testing in breast Cancer: American Society of Clinical Oncology/College of American Pathologists Clinical Practice Guideline Update. J Clin Oncol. 2013; 31(31):3997-4013.

24. Andrews S. FastQC: a quality control tool for high throughput sequence data. 2010. Available from: https://www.bioinformatics.babraham.ac.uk/ projects/fastqc

25. Haeussler M, Zweig AS, Tyner C, Speir ML, Rosenbloom KR, Raney BJ, et al. The UCSC genome browser database: 2019 update. Nucleic Acids Res. 2019; 47(D1):D853-8.

26. Wingett $S$, Andrews S. FastQ screen: a tool for multi-genome mapping and quality control. F1000Research. 2018;7:1338.

27. Nickes D, Sandmann T, Ziman R, Bourgon R. NanoStringQCPro: Quality metrics and data processing methods for NanoString mRNA gene expression data. 2018. Available from: https://bioconductor.org/packages/ release/bioc/html/NanoStringQCPro.html

28. R Core Team. R: a language and environment for statistical computing. Vienna: R Foundation for statistical Computing; 2017.

29. Shrout PE, Fleiss JL. Intraclass correlations: uses in assessing rater reliability. Psychol Bull. 1979;86(2):420-8. 